\title{
TOMOGRAFIA COMPUTADORIZADA EM DECUBITO VENTRAL E LATERAL NO DIAGNOSTICO DIFERENCIAL ENTRE HEMATOMA SUBDURAL E ATROFIA CORTICAL
}

\author{
EDUARDO BIANCO \\ MILTON $K$. SHIBATA \\ GILBERTO MACHADO DE ALMEIDA
}

O diagnóstico diferencial tomográfico (TC) entre atrofia cerebral e efusão subdural, em crianças, nem sempre é fácil, especialmente quando há acúmulo de líquido nas regiões frontais. Nestes casos temos realizado tomografias em decúbito ventral ou lateral, além do exame estandard. Isto tem a finalidade de detectar mudanças na posição relativa do encéfalo e mudança na forma dos sulcos e giros, características de atrofia cerebral.

Na figura 1-A, em decúbito ventral, nota-se grande aumento do espaço subaracnóideo frontal e atrofia cerebral. Na figura 1-B, em decúbito ventral, os sulcos e giros são melhor visibilizados e a fissura interhemisférica está mais larga.

$\mathrm{Na}$ figura 2 estas alterações são mais evidentes. Os sulcos, giros e espaço subaracnóideo na região occipital tornam-se mais evidentes na tomografia com a criança em decúbito lateral esquerdo (fig. 2-B), do que em decúbito dorsal (fig. 2-A). No caso seguinte (fig. 3), também de atrofia cerebral, nota-se, com a mudança de decúbito, alteração do espaço subaracnóideo.

Nas coleções subdurais, o espaço extracerebral não se modifica de maneira significante, com o decúbito. As figuras 4 e 5 mostram as tomografias de uma criança com coleção subdural pós-meningite (4-A e 4-B) e de outras criança com hematoma subdural crônico (5-A e 5-B). Com o decúbito lateral, o aspecto de espaço extracerebral praticamente não se modificou.

Julgamos que este método, isto é, a realização de tomografias em outras posições além da usual, pode ser útil para esclarecer casos de diagnóstico duvidoso.

\section{RESUMO}

A realização da tomografia computadorizada em decúbito lateral ou ventral, pode ser útil no diagnóstico diferencial entre atrofia cerebral e coleção subdural. Alguns casos são apresentados.

Clínica Neurocirúrgica e Centro de Tomografia do Hospital 9 de Julho, São Paulo, Brasil. 

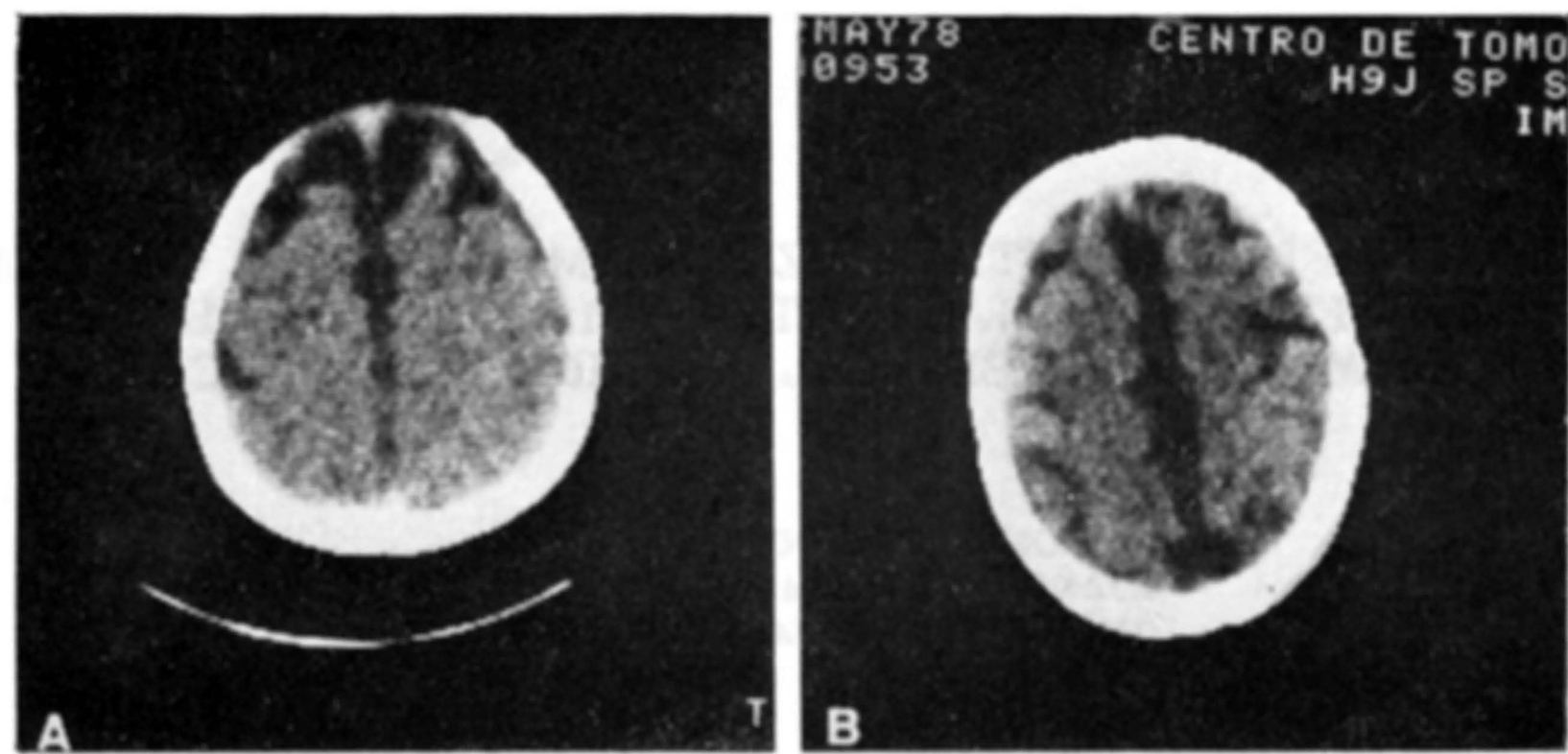

Fig. I - Atrofia cerebral. TC om decubito dorsal (A) e ventral (B) mostrando mostrando as modificagoes do espago subaracnoideo
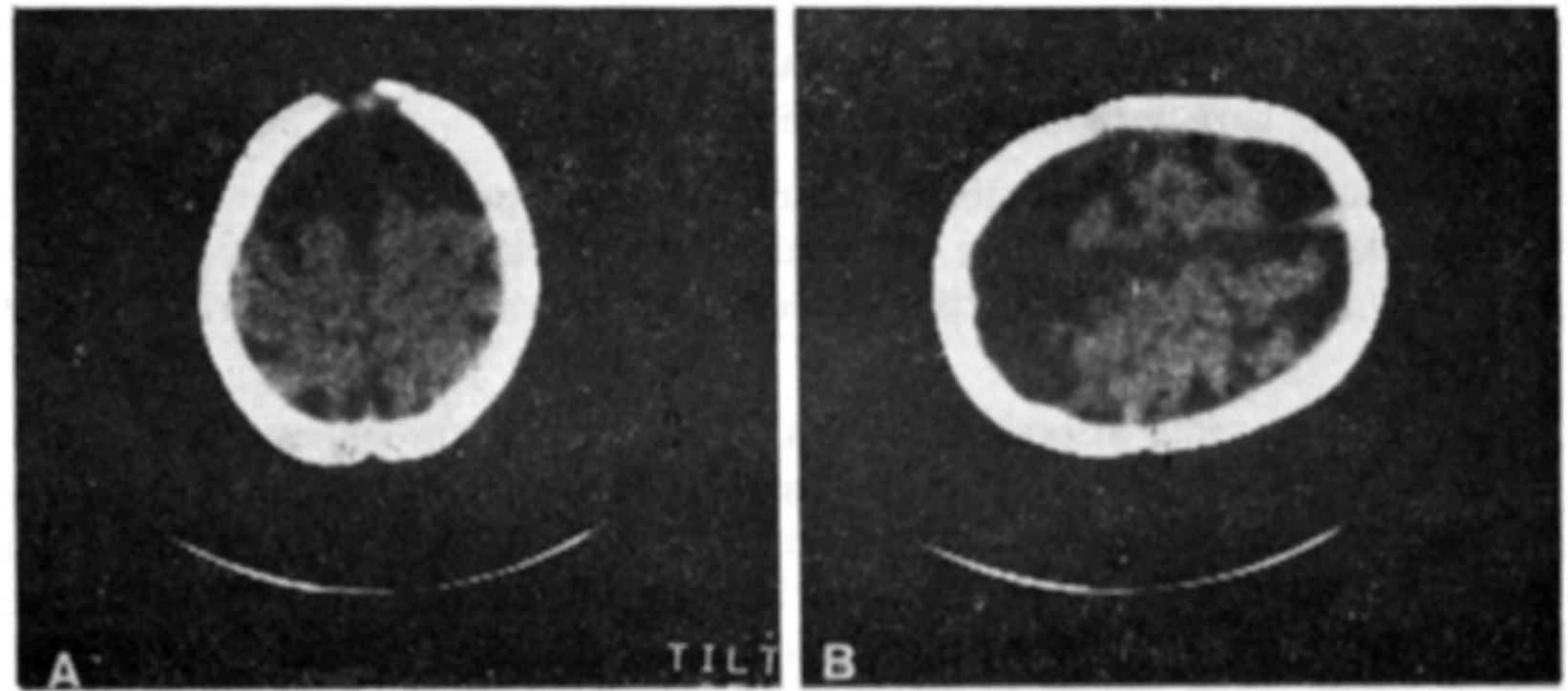

Fig. 8 - Atrofia cerebral. TC em decabito dorsal (A) e lateral osquerdo (B) mostrando as alteragdes do espaco subaracnoideo.
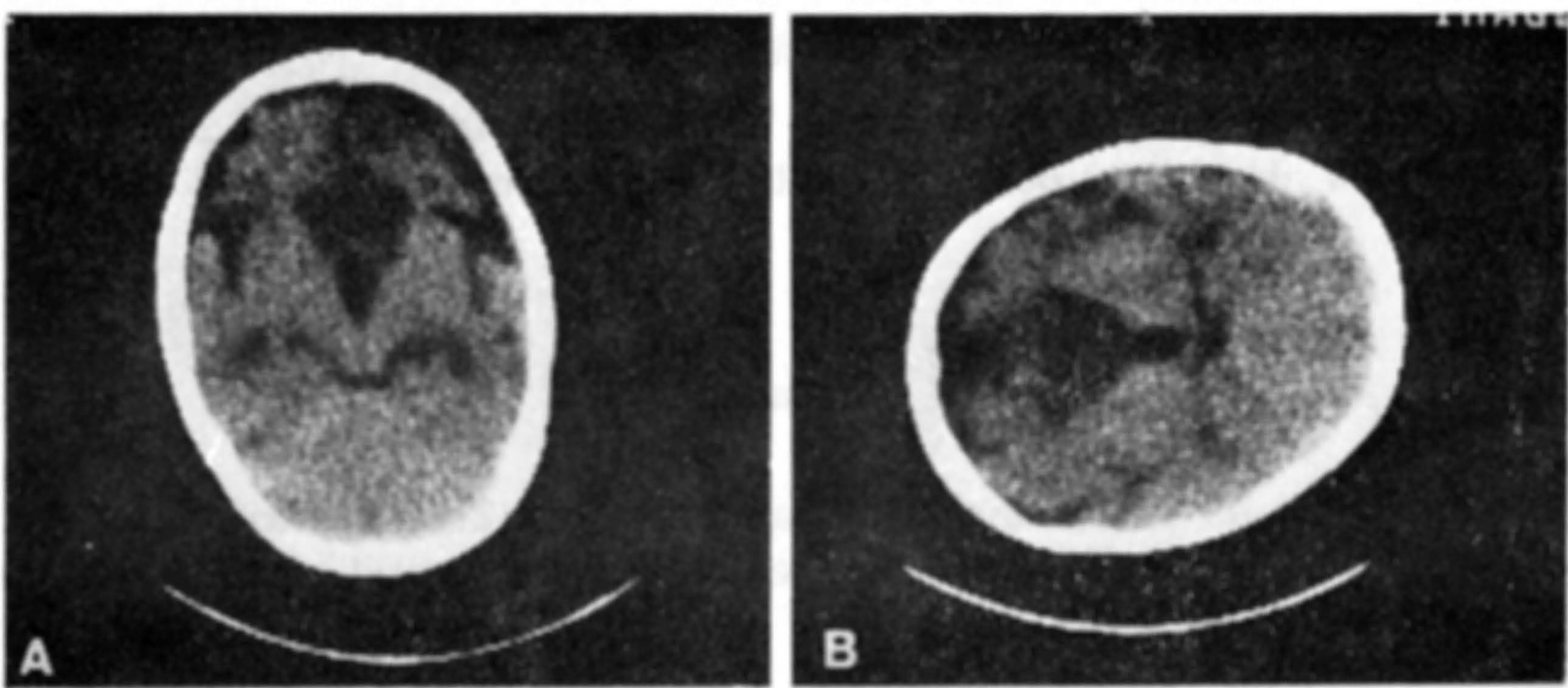

Fig. 3 - Atrofia cerebral. TC um decubito dorsal (A) a lateral esquerdo (B) notando-se as diferengas do espaso subaraonbideo. 

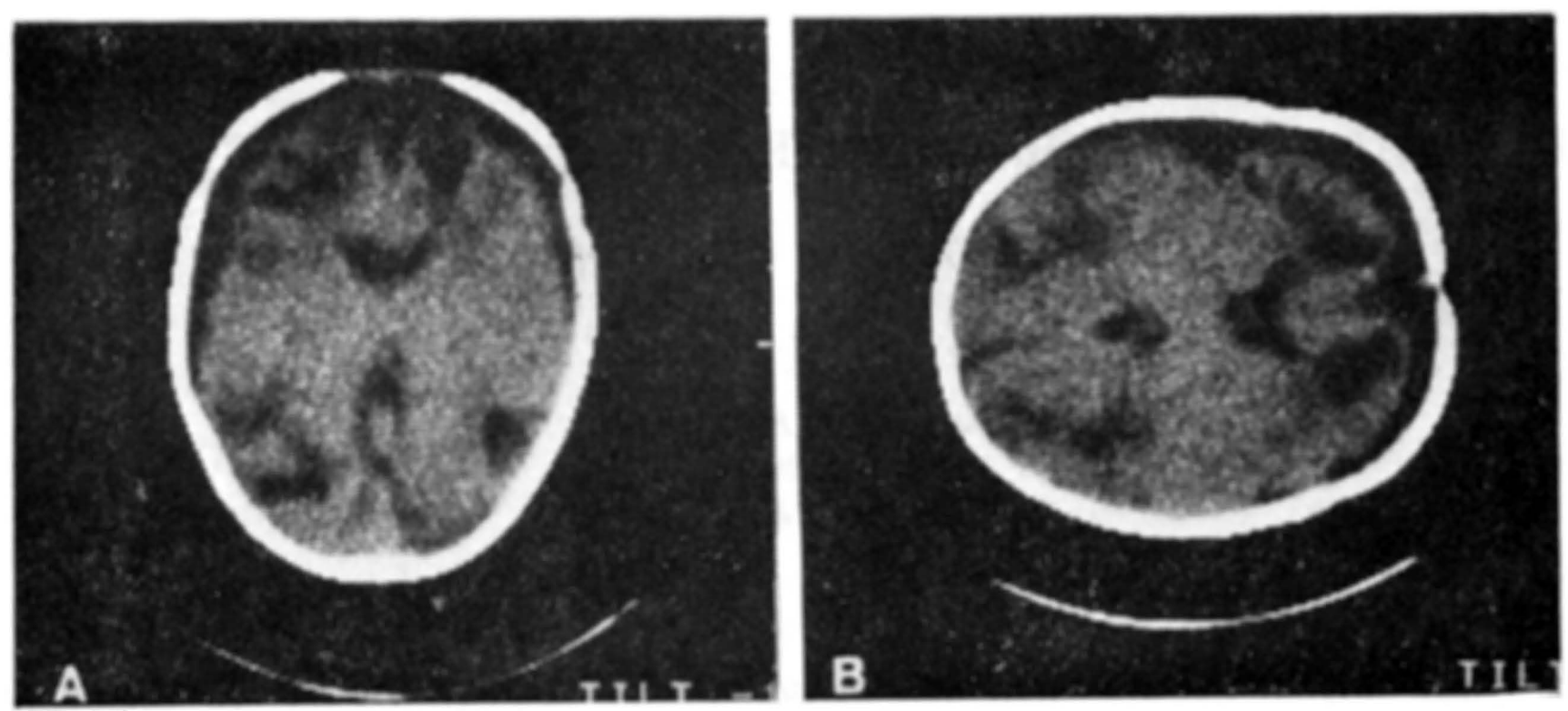

Fig. 4 Coleço subdural pos-mexingite. 0 espaço extracerebral ndo se modifica nos diferentes decibitos.
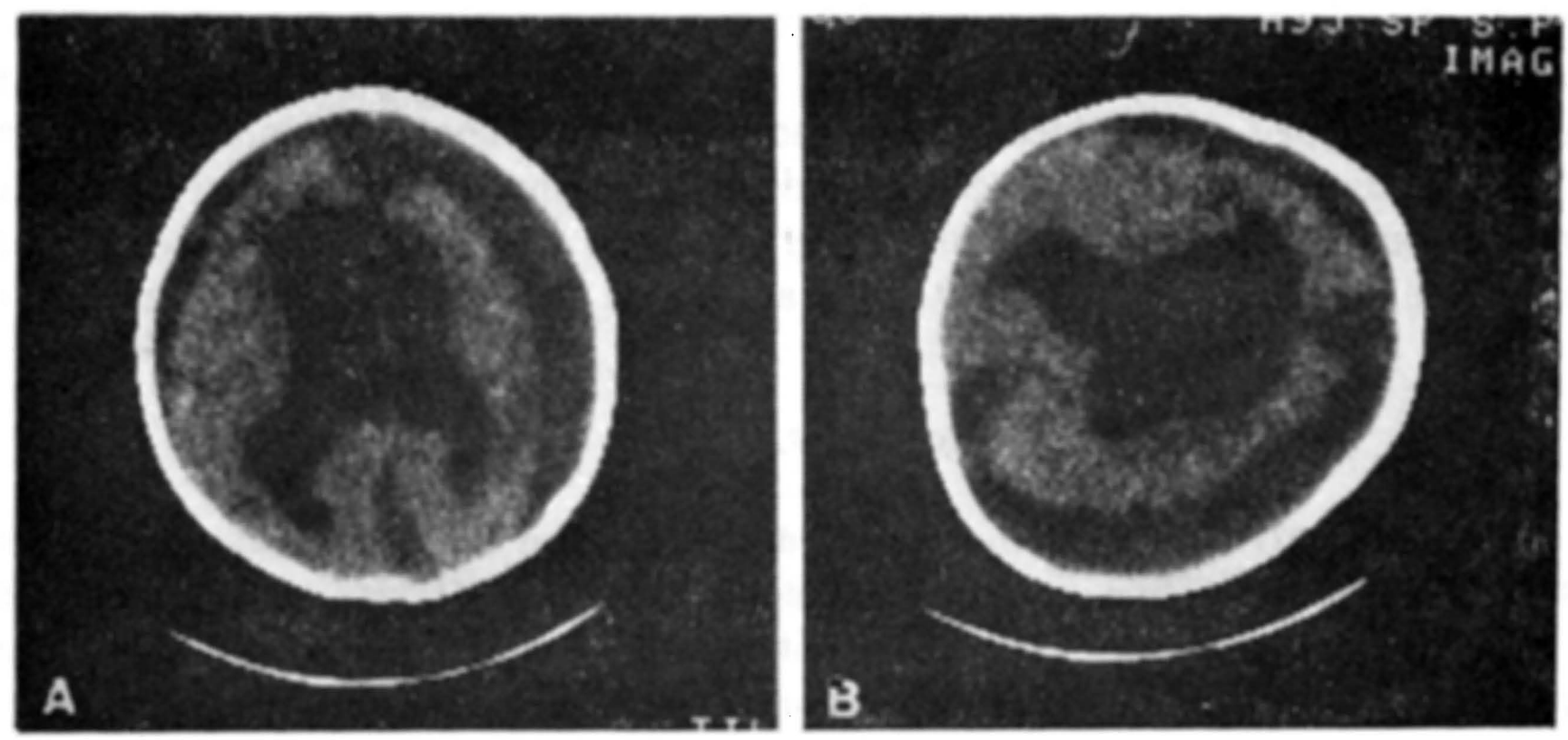

F'ig. 5 - Hematoma subdural cronico. I'C em decubito dorsal (A) e lateral atresto (B). O espaco extracircbral permanece praticamente inalterado.

\section{SUMMARY}

Computer assisted tomography in lateral and prone position in the differencial diagnosis between brain atrophy and subdural colections.

The differencial diagnosis between brain atrophy versus subdural effusion in children has shown some difficulties in a few cases, mainly when the frontal subarachnoid space is very large on computed tomography. We think that a simple procedure, that is, CT scans in the lateral and prone position followirg the standard examination, may be worth to clarify those cases of doultfoull diagnosis. 